\title{
IUFOST2006/1031
}

\section{From Molecular to Macroscopic Investigation of Food Structure us- ing NMR and MRI Techniques}

\author{
F. Mariette \\ Cemagref, Food process engineering research unit, CS 64427, 17 avenue de Cucillé, 35044 Rennes, France \\ francois.mariette@cemagref.fr
}

\begin{abstract}
Magnetic resonance imaging (MRI) has been used as a powerful technique for medical diagnostic probe of the human body. Since, the range of MRI application has been extended to food science. Food display an enormous range of structural and compositional complexity and heterogeneity which explained the sensory and safety properties. Then, the investigation of such products require the use of non invasive techniques providing quantitative information on the spatial organization. From NMR parameters such as proton density and relaxation time parameters, many food characteristics could be quantified. For example, distribution of the chemical composition, such as water and fat distribution in food and animal products such as pig carcasses and flesh of trout could be quantified from proton density and also from the relaxation time parameters. However, the sensitivity of the relaxation parameters to both rotational and transversal molecular mobility offers a unique way to evaluate the effect of processing operations on food structure at a molecular and microscopic length scale. For example, structural modifications from macromolecules induced during maturation, cooking, frying and freezing-thawing operations can be assessed non invasively and spatial variation can be evaluated. Moreover, water exchanges by diffusion between neighboring water compartments induce strong variation in the water relaxation decay curve. These phenomena is widely used for the study of fruit and vegetable structure at a cellular length scale. Indeed, all factors which will induce a change of the cell integrity could be monitor from MRI. Other examples of MRI application come from the links between NMR parameters and physical parameters such as temperature flow and diffusion. Therefore, from specific MRI measurement protocols heat transfer, flow and diffusion behaviors open new opportunities to fascinating challenges in food sciences. The objective of this presentation will be to show how complementary studies including both NMR relaxometry and MRI have expanded the number of applications of MRI in food research.
\end{abstract}

